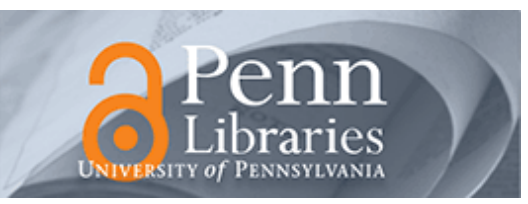

University of Pennsylvania

ScholarlyCommons

$1-11-2013$

\title{
Relationship Between Neighbor Number and Vibrational Spectra in disordered colloidal clusters with attractive interactions
}

\author{
Peter J. Yunker \\ University of Pennsylvania \\ Zexin Zhang \\ Soochow University \\ Matthew Gratale \\ University of Pennsylvania \\ Ke Chen \\ Chinese Academy of Sciences \\ Arjun G. Yodh \\ University of Pennsylvania, yodh@phsyics.upenn.edu \\ Follow this and additional works at: https://repository.upenn.edu/physics_papers \\ Part of the Physics Commons
}

\section{Recommended Citation}

Yunker, P. J., Zhang, Z., Gratale, M., Chen, K., \& Yodh, A. G. (2013). Relationship Between Neighbor Number and Vibrational Spectra in disordered colloidal clusters with attractive interactions. Retrieved from https://repository.upenn.edu/physics_papers/276

Yunker, P. J., Zhang, Z, Gratale, M., Chen, K., \& Yodh, A. G. (2013). Relationship between neighbor number and vibrational spectra in disordered colloidal clusters with attractive interactions. Journal of Chemical Physics, 138(12), 12A525. doi: 10.1063/1.4774076

(C) 2013 American Institute of Physics. This article may be downloaded for personal use only. Any other use requires prior permission of the author and the American Institute of Physics.

This paper is posted at ScholarlyCommons. https://repository.upenn.edu/physics_papers/276

For more information, please contact repository@pobox.upenn.edu. 


\title{
Relationship Between Neighbor Number and Vibrational Spectra in disordered colloidal clusters with attractive interactions
}

\begin{abstract}
We study connections between vibrational spectra and average nearest neighbor number in disordered clusters of colloidal particles with attractive interactions. Measurements of displacement covariances between particles in each cluster permit calculation of the stiffness matrix, which contains effective spring constants linking pairs of particles. From the cluster stiffness matrix, we derive vibrational properties of corresponding "shadow" glassy clusters, with the same geometric configuration and interactions as the "source" cluster but without damping. Here, we investigate the stiffness matrix to elucidate the origin of the correlations between the median frequency of cluster vibrational modes and average number of nearest neighbors in the cluster. We find that the mean confining stiffness of particles in a cluster, i.e., the ensemble-averaged sum of nearest neighbor spring constants, correlates strongly with average nearest neighbor number, and even more strongly with median frequency. Further, we find that the average oscillation frequency of an individual particle is set by the total stiffness of its nearest neighbor bonds; this average frequency increases as the square root of the nearest neighbor bond stiffness, in a manner similar to the simple harmonic oscillator.

\section{Disciplines}

Physical Sciences and Mathematics | Physics

\section{Comments}

Yunker, P. J., Zhang, Z, Gratale, M., Chen, K., \& Yodh, A. G. (2013). Relationship between neighbor number and vibrational spectra in disordered colloidal clusters with attractive interactions. Journal of Chemical Physics, 138(12), 12A525. doi: 10.1063/1.4774076

(C) 2013 American Institute of Physics. This article may be downloaded for personal use only. Any other use requires prior permission of the author and the American Institute of Physics.
\end{abstract}




\title{
Relationship between neighbor number and vibrational spectra in disordered colloidal clusters with attractive interactions
}

\author{
Peter J. Yunker, ${ }^{1}$ Zexin Zhang, ${ }^{2,3}$ Matthew Gratale, ${ }^{1}$ Ke Chen, ${ }^{4}$ and A. G. Yodh ${ }^{1}$ \\ ${ }^{1}$ Department of Physics and Astronomy, University of Pennsylvania, Philadelphia Pennsylvania 19104, USA \\ ${ }^{2}$ Center for Soft Condensed Matter Physics and Interdisciplinary Research, Soochow University, \\ Suzhou 215006, China \\ ${ }^{3}$ Complex Assemblies of Soft Matter, CNRS-Rhodia-UPenn UMI 3254, Bristol, Pennsylvania 19007, USA \\ ${ }^{4}$ Beijing National Laboratory for Condensed Matter Physics and Key Laboratory of Soft Matter Physics, \\ Institute of Physics, Chinese Academy of Sciences, Beijing 100190, China
}

(Received 17 October 2012; accepted 18 December 2012; published online 11 January 2013)

\begin{abstract}
We study connections between vibrational spectra and average nearest neighbor number in disordered clusters of colloidal particles with attractive interactions. Measurements of displacement covariances between particles in each cluster permit calculation of the stiffness matrix, which contains effective spring constants linking pairs of particles. From the cluster stiffness matrix, we derive vibrational properties of corresponding "shadow" glassy clusters, with the same geometric configuration and interactions as the "source" cluster but without damping. Here, we investigate the stiffness matrix to elucidate the origin of the correlations between the median frequency of cluster vibrational modes and average number of nearest neighbors in the cluster. We find that the mean confining stiffness of particles in a cluster, i.e., the ensemble-averaged sum of nearest neighbor spring constants, correlates strongly with average nearest neighbor number, and even more strongly with median frequency. Further, we find that the average oscillation frequency of an individual particle is set by the total stiffness of its nearest neighbor bonds; this average frequency increases as the square root of the nearest neighbor bond stiffness, in a manner similar to the simple harmonic oscillator. () 2013 American Institute of Physics. [http://dx.doi.org/10.1063/1.4774076]
\end{abstract}

\section{INTRODUCTION}

Colloidal particles that interact via strong short-range attractive potentials form disordered clusters, ${ }^{1,2}$ and these clusters often have large local packing fractions and particle configurations similar to common "space-filling" colloidal glasses. Recent experiments ${ }^{3}$ have suggested that the vibrational properties of small clusters of colloidal particles with attractive interactions are similar to the vibrational properties of jammed glasses. ${ }^{4,5}$ Specifically, the median frequency of the cluster vibrational spectrum, $\omega_{\text {Med }}$, was found to correlate strongly with the average number of neighbors, $\overline{N N}$, in the cluster. This behavior resembles that in simulations of mechanically stable athermal jammed packings, wherein the socalled Boson peak frequency scales linearly with the average number of contacts between particles. ${ }^{5}$ Thus, further exploration of the strong correlation between $\overline{N N}$ and $\omega_{\text {Med }}$ holds potential to distinguish properties universal to disordered systems (e.g., Refs. 3 and 5) from properties that are system specific (e.g., Ref. 6).

In this contribution we experimentally investigate the vibrational properties of disordered clusters of colloidal particles with attractive interactions. Disordered clusters are formed in water-lutidine suspensions wherein wetting effects induce fluid mediated attraction between micron-sized polystyrene particles. ${ }^{6-9}$ Each cluster is characterized by the number of particles it contains $(\mathrm{N})$ and its average number of nearest neighbors $(\overline{N N})$. Displacement covariance matrix techniques employed in recent papers ${ }^{10-13}$ are then used to determine phonon spectra of each attractive glassy cluster. Specifically, video microscopy is employed to measure displacement covariances between each particle pair in each cluster. Using this information, we calculate the cluster stiffness matrix, $K_{i j}$, which contains the effective spring constants that link each pair of particles $i$ and $j$. From the stiffness matrix, we derive the phonon density of states of corresponding "shadow" attractive glass clusters with the same geometric configuration and interactions as the "source" experimental colloidal system, but absent damping. ${ }^{11}$ While numerical simulations can also access the vibrational density of states of disordered systems (e.g., Ref. 5), the results presented here are from real clusters that self-assembled via complex interactions or were assembled with laser tweezers. Thus, these results are relevant for real systems that can easily be made in the lab, as opposed to idealized model systems.

Previous work has demonstrated that the median cluster vibrational frequency, $\omega_{\text {Med }}$, depends strongly on the average number of nearest neighbors, $\overline{N N}$, but weakly on the number of particles, $N$, in the cluster, and weakly on other structural parameters of the cluster. ${ }^{3}$ The present experiments reveal that $\omega_{\text {Med }}$ has an even stronger correlation with the mean stiffness felt by individual particles than with $\overline{N N}$, i.e., the mean of the diagonal elements of the stiffness matrix, $\bar{K}_{i i}$ compared to $\overline{N N} . \bar{K}_{i i}$, in turn, is shown to be correlated with $\overline{N N}$, among other factors. This experimental observation is further supported by a simple theoretical argument. In addition, we find that the average oscillation frequency of any given particle in the cluster increases as the square root of its total nearest 
neighbor bond stiffness, similar to the behavior of a simple harmonic oscillator. Thus, while global spectral properties are controlled, in large part, by network connectivity, local vibrational properties are controlled by local stiffness. Finally, the work provides microscopic evidence that spectroscopic observations can be used to probe structural coordination properties of disordered media.

\section{BACKGROUND}

\section{A. Experimental system}

The experiments employ bidisperse suspensions of micron-sized polystyrene particles (Invitrogen), with diameters of $d_{S}=1.5 \mu \mathrm{m}$ and $d_{L}=1.9 \mu \mathrm{m}$, and number ratio of $1: 2$, respectively. Binary mixtures of particles were used to minimize crystallization. Particles were suspended in a mixture of water and 2, 6-lutidine (WL) near its critical composition, i.e., a lutidine mass fraction of 0.28 . Colloidal particles in this near-critical WL binary mixture experience temperature dependent fluid-mediated repulsive or attractive interactions. ${ }^{7,8}$ Particles are well dispersed at $T=300.15 \mathrm{~K}$, but at $T$ $=306.45 \mathrm{~K}$ they aggregate and form clusters, because lutidine preferentially wets the polystyrene particles.

A plethora of different disordered particle clusters are created by first suspending particles deep in the repulsive regime $(300.15 \mathrm{~K})$, and then rapidly increasing the sample temperature (to 306.5) in situ. ${ }^{3}$ Sample temperature control was accomplished using an objective heater (Bioptechs) connected to the microscope oil immersion objective. ${ }^{14-16}$ Particles are confined between two glass coverslips (Fisher) with a spacing of $\sim 1.1 \pm 0.05 d_{L}$; the sample is therefore a quasi-2D system. The glass cell was treated with hexamethyldisilazane (HMDS), so the particle-wall interaction potential is repulsive at relevant temperatures. ${ }^{9}$ The global area fraction is $\sim 0.2$.

Disordered clusters of various sizes and shapes selfassemble. Other clusters are created with aid of laser tweezers, ${ }^{17}$ either by grabbing particles and adding them to existing clusters, or by dragging an optical trap across a cluster and forcing rearrangements. Samples equilibrated for about six hours before measurements began. Video data were collected at a rate of 10 frames per second.

The number of particles, $N$, is only weakly correlated with $\overline{N N}$ (Fig. 1). For linear clusters $(\overline{N N}<2)$, $N$ and $\overline{N N}$ have a strong correlation; for more compact clusters $(\overline{N N} \geq 2), N$ and $\overline{N N}$ have a very weak correlation.

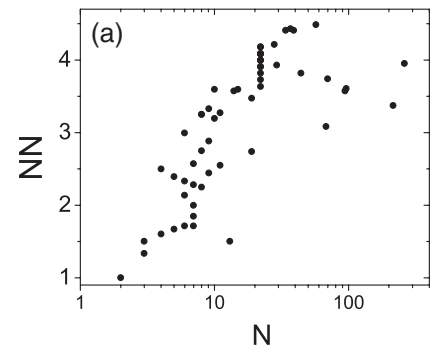

(b)

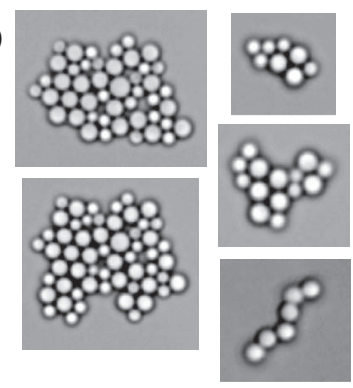

FIG. 1. (a) Plot summarizing the number of particles, $\mathrm{N}$, and average number of nearest neighbors, $\overline{N N}$, in every cluster studied. (b) Experiment snapshots of five different clusters.
Note, Fig. 1(a) is plotted on a linear-log plot. Thus, while it appears $N$ and $\overline{N N}$ may be strongly correlated for $N<20$, this is not the case (for $N<20, R^{2}=0.37$ ).

\section{B. Theoretical analysis}

The vibrational properties of each cluster are extracted by measuring displacement correlations of the particles within each cluster. Specifically, we define $u(t)$ as the $2 N$-component vector of the displacements of all particles from their average positions $(\bar{x}, \bar{y})$, and we extract the time-averaged displacement correlation matrix (covariance matrix), $C_{i j}=\left\langle u_{i} u_{j}\right\rangle_{t}$, from experiment, where $i, j=1, \ldots, 2 N$ run over all particle and positional coordinates, and the average runs over time. Note, the calculation of $C_{i j}$ depends only on particle displacements. In the harmonic approximation, the correlation matrix is directly related to the cluster stiffness matrix, defined as the matrix of second derivatives of the effective pair interaction potential with respect to particle position displacements. In particular, $\left(C^{-1}\right)_{i j} k_{B} T=K_{i j}$, where $K_{i j}$ is the stiffness matrix. Experiments that measure $C$ therefore permit us to construct and derive properties of a "shadow" glassy cluster that has the same static properties as our colloidal system (e.g., same correlation matrix, same stiffness matrix, etc.). ${ }^{1-13}$ Following Ref. 18, we expect undamped particles, that repel at very short-range and attract on longer length scales, due to fluid mediated effects, to give rise to solid-like vibrational behavior on time scales long compared to particle collision times, but short compared to the time between particle rearrangement events..$^{10,13}$

The stiffness matrix is directly related to the dynamical matrix characterizing system vibrations, $D_{i j}=\frac{K_{i j}}{m_{i j}}$, where $m_{i j}=\sqrt{m_{i} m_{j}}$ and $m_{i}$ is the mass of particle $i$. The eigenvectors of the dynamical matrix correspond to particle displacement amplitudes associated with the various phonon modes, and the eigenvalues of the dynamical matrix are the frequencies/energies of the corresponding modes. Data were collected for $10000 \mathrm{~s}$ so that the number of degrees of freedom, $8 \leq 2 N \leq 500$, is small compared to the number of time frames $(>10 \times 2 N)$. Additionally, we find $K_{i j}$ is far above the noise only for adjacent particles, as expected.

\section{Finite-sampling correction}

To correct for finite-time effects, we extrapolate to "infinite time" and derive "true" frequencies. To this end, we use the relationship $1 / \omega\left(N_{\text {frames }}\right)=1 / \omega(\mathrm{inf})+m 2 N / N_{\text {frames }}$, where $N_{\text {frames }}$ is the number of images collected, $2 N$ is the number of degrees of freedom in the sample, and $m$ is a multiplicative constant (Fig. 2). ${ }^{19}$ This correction was found to have little effect on $\omega_{\text {Med }}$; i.e., the coefficient of determination, $R^{2}=0.85$, was unchanged when comparing corrected and uncorrected frequencies.

As a side note, the correlation does not depend on particle size. We plotted the median frequency versus the average number of neighbors for large particles and the average number of neighbors for small particles. The correlation persists, but is weaker. The best linear fits for $N N>2$ yield $R^{2}=0.80$ and $R^{2}=0.67$ for small and large particles, 


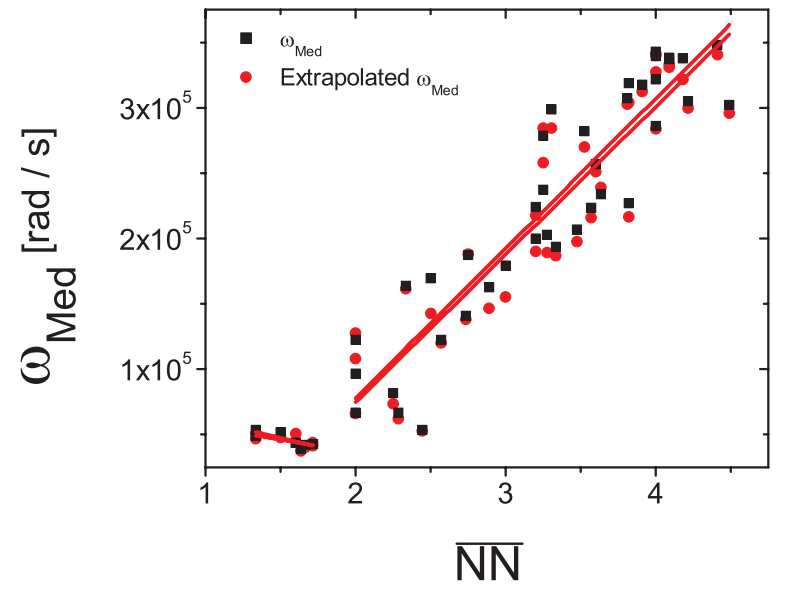

FIG. 2. The median frequency, $\omega_{\text {Med }}$ (black squares), and the infinite-timeextrapolated median frequency (red circles), are plotted versus the average number of nearest neighbors, $\overline{N N}$.

respectively. Thus, the correlation is at its strongest when both large and small particles are considered.

\section{RESULTS}

\section{A. Effect of $\overline{K_{i i}}$ on $\omega_{M e d}$}

Interestingly, that data reveal that a strong relationship exists between $\omega_{\text {Med }}$ and $\overline{N N}$, despite the fact that $\overline{N N}$ does not directly enter into any calculation of $\omega_{\text {Med }}$ (Fig. 2). Thus, information about $\overline{N N}$ must be implicit in $K_{i j}$. In fact, the average of the diagonal elements in the stiffness matrix, $\bar{K}_{i i}$, has a strong correlation with $\overline{N N}$ too (Fig. 3). This effect might be expected, since the diagonal elements of the stiffness matrix typically balance out the springs of neighbors pushing against the selected particle $i$, i.e., $\sum_{j \neq i} K_{i j} \approx-K_{i i}$. Here, each spring represents the strength of the harmonic interaction between particle $i$ and particle $j$, and $K_{i i}$ is essentially the curvature of the harmonic potential well that confines particle $i$. Since the sum is dominated by the nearest neighbor springs (i.e., nonnearest neighbor spring constants are approximately zero), $K_{i i}$ depends strongly on the neighbor number of particle $i$. Thus, as $\overline{N N}$ increases, $\overline{K_{i i}}$ tends to increase as well, i.e., more neighbors lead to stronger confinement. $\overline{K_{i i}}$ can also increase

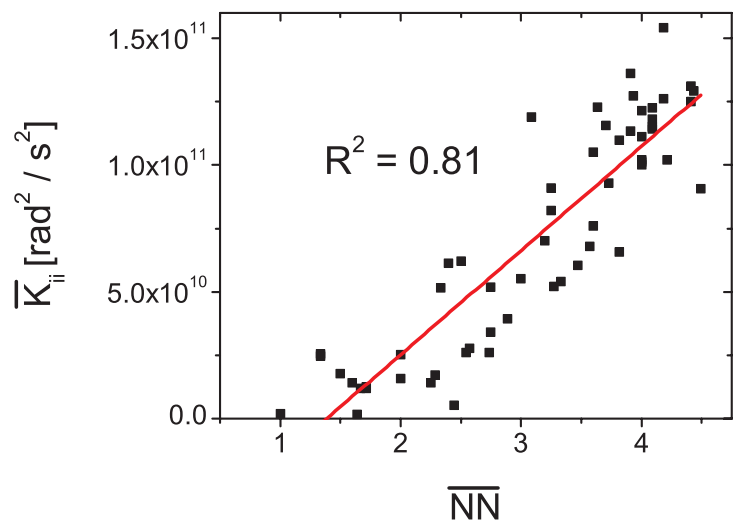

FIG. 3. The mean of the diagonal value of the stiffness matrix, i.e., $\bar{K}_{i i}$, plotted versus the average number of nearest neighbors in the cluster, $\overline{N N}$.

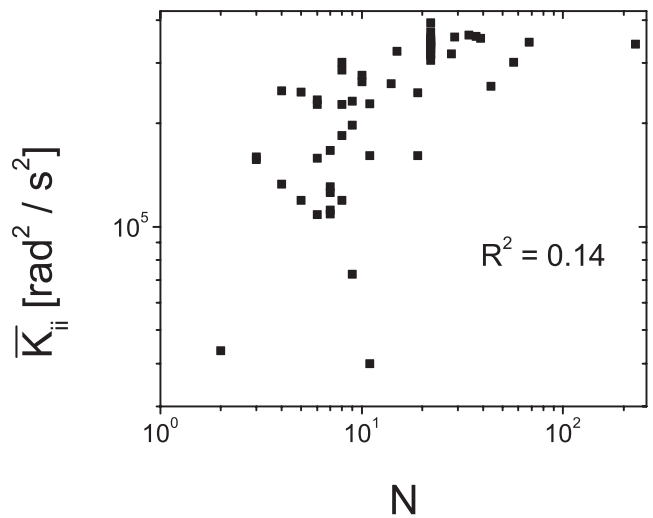

FIG. 4. The mean of the diagonal value of the stiffness matrix, i.e., $\bar{K}_{i i}$, plotted versus the total number of particles in the cluster, $N$.

(or decrease) without changing average $\overline{N N}$, for example via changes of the interparticle separation between neighboring particles.

Conversely, $\overline{K_{i i}}$ has relatively little correlation with the total number of particles in a cluster, $N$ (see Fig. 4). This weak correlation $\left(R^{2}=0.14\right)$ is expected, since non-nearestneighbor spring constants are $\sim 0$, and the sum $\sum_{j \neq i} K_{i j}$ only depends strongly on $\overline{N N}$.

We next investigated the relationship between $\bar{K}_{i i}$ and $\omega_{\text {Med }}$. The correlation between these two parameters is very strong; the coefficient of determination is $R^{2}=0.92$ (Fig. 5 inset). In fact, this correlation is even stronger than the one observed between $\omega_{\text {Med }}$ and $\overline{N N}$ (Fig. 2). Finally, we investigated the correlation between $\sqrt{\bar{K}_{i i}}$ and $\omega_{\text {Med }}$ (Fig. 5), since $K_{i j} e_{i}=\omega^{2} e_{i}$, where $e_{i}$ is the polarization of mode $i$. The correlation between these two parameters is also very strong, with a coefficient of determination $R^{2}=0.93$, and the best linear fit of $\sqrt{\bar{K}_{i i}}$ and $\omega_{\text {Med }}$ gives $\omega_{\text {Med }} \propto 1.09(4) \sqrt{\bar{K}_{i i}}$. The latter observation implies that $\sqrt{\overline{K_{i i}}}$ and $\omega_{\text {Med }}$ increase with a nearly 1-to-1 ratio; note, however, the accessible dynamic range is not large enough for a power-law fit to unambiguously distinguish between $\omega_{M e d} \propto \bar{K}_{i i}$ and $\omega_{M e d} \propto \sqrt{\bar{K}_{i i}}$, even though a very strong correlation exists.

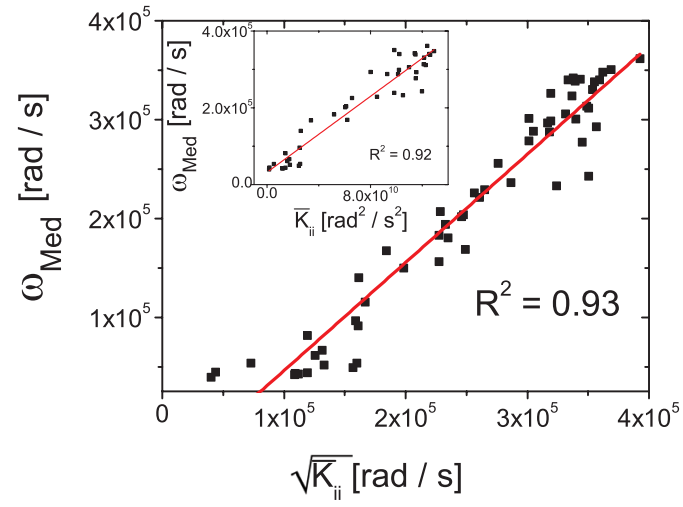

FIG. 5. The median vibrational frequency of cluster mode spectra versus the square root of the mean of the diagonal value of the cluster stiffness matrix, i.e., $\sqrt{\bar{K}_{i i}}$. Black squares and red circles represent clusters with $\overline{N N} \geq 2$ and $\overline{N N}<2$, respectively. Inset: The median vibrational frequency of cluster mode spectra versus the mean diagonal value of the cluster stiffness matrix, i.e., $\bar{K}_{i i}$. 
The strong correlation between $\sqrt{\overline{K_{i i}}}$ and $\omega_{\text {Med }}$ is selfconsistent with the fact that $\omega_{\text {Med }}$ is largely independent of $N$; $\bar{K}_{i i}$ is strongly correlated with $\overline{N N}$ (Fig. 3), but only weakly correlated with $N$ (Fig. 4). Physically, increasing the number of nearest neighbors increases the number of bonds in a particle's confining "cage," and is a factor that strongly affects $K_{i i}$. However, the average number of neighbors in a cluster is only weakly correlated with the number of particles in a cluster, $N$ (Fig. 1). A simple theoretical derivation of the correlation between $\sqrt{\bar{K}_{i i}}$ and $\omega_{\text {Med }}$ is provided in the Appendix. In essence, $K_{i j}$ can formally be written as the sum of a matrix, $A_{i j}$, and the identity matrix multiplied by $\overline{K_{i i}}$. The median frequency of $K_{i j}$ is then the median frequency of $A_{i j}$ plus $\overline{K_{i i}}$; thus, $\omega_{\text {Med }} \propto \overline{K_{i i}}$.

As a side note, the correlation between $\omega_{\text {Med }}$ and $\overline{K_{i i}}$ does not depend on particle size. We plotted the median frequency versus the average number of neighbors for large particles and the average number of neighbors for small particles. The correlation persists, but is weaker. The best linear fits for $N N>2$ yield $R^{2}=0.60$ and $R^{2}=0.82$ for small and large particles, respectively. Thus, the correlation is at its strongest when both large and small particles are considered.

Finally, we note that the previously observed ${ }^{3}$ relationship between $\omega_{\text {Med }}$ and $N N$ had two regimes, suggesting a clear distinction between the effects of locally rigid structures $(\overline{N N}>2)$ and purely floppy structures $(\overline{N N}<2)$. In the present experiment, however, the observed relationship between $\bar{K}_{i i}$ and $\omega_{\text {Med }}$, does not clearly separate into two regimes and thus does not readily distinguish between the effects of these qualitatively different structures. This scenario arises partially due to the limitations of calculating a meaningful median vibrational frequency. For clusters with $\overline{N N}<2$, more than half of the modes are floppy. These modes have small frequencies, independent of $\bar{K}_{i i}$, which are nearly zero. Thus, the median frequency for these clusters does not depend on $\bar{K}_{i i}$.

\section{B. Vibrational properties of individual particles}

To explore local vibrational properties around each particle, we calculate the polarization vector-weighted frequency $\left\langle\omega_{i \alpha}\right\rangle=\sum_{j=1 . .2 N} \omega(j) * e(j)_{i}^{2} / \sum_{j=1 . .2 N} e(j)_{i \alpha}^{2}$, where $e(j)_{i \alpha}^{2}$ is the polarization vector for mode $j$, particle $i$, direction $\alpha(x$ or $y)$. Essentially, $\left\langle\omega_{i \alpha}\right\rangle$ measures the average frequency in which particle $i$ participates in direction $\alpha .\left\langle\omega_{i \alpha}\right\rangle$ has a strong correlation with $K_{i i}$ (Fig. 6). Further, the best power-law fit is $\left\langle\omega_{i \alpha}\right\rangle \propto K_{i i}^{0.47(1)}$, reminiscent of a simple harmonic oscillator, for which $\omega \propto \sqrt{K}$.

Surprisingly, we find that the location of a particle to have little effect on $\left\langle\omega_{i \alpha}\right\rangle$, i.e., $\left\langle\omega_{i \alpha}\right\rangle$ is not qualitatively different for particles on the cluster surface as compared to particles in the cluster interior. In either case, $\left\langle\omega_{i \alpha}\right\rangle$ simply depends on $K_{i i}$.

\section{DISCUSSION AND SUMMARY}

We have found that the median vibrational frequency, $\omega_{M e d}$, of a disordered cluster is predominantly set by $\bar{K}_{i i}$, the ensemble-averaged confining stiffness for particles in the

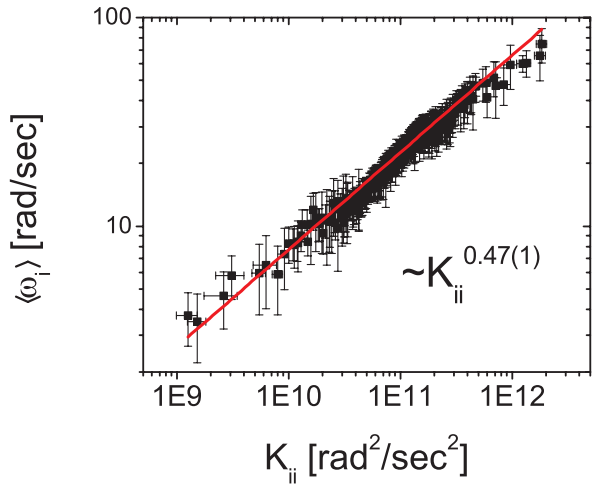

FIG. 6. The eigenvector-weighted frequency, $\left\langle\omega_{i \alpha}\right\rangle$, plotted versus diagonal stiffness matrix elements, $K_{i i}$. Solid red line is the best power-law fit. The relationship between $\left\langle\omega_{i \alpha}\right\rangle$ and $K_{i i}$ is reminiscent of a simple harmonic oscillator.

cluster. $\bar{K}_{i i}$, in turn, has a strong correlation with $\overline{N N}$, the average number of nearest neighbors; this latter effect produces the previously observed ${ }^{3}$ correlations between $\overline{N N}$ and $\omega_{\text {Med }}$. Thus, the present observations suggest that the fundamental origin between $\overline{N N}$ and $\omega_{\text {Med }}$ is a result of mean confining stiffness in the cluster. Further, the average frequency of an individual particle, $\left\langle\omega_{i \alpha}\right\rangle$, increases as a power-law with $K_{i i}$, i.e., $\left\langle\omega_{i \alpha}\right\rangle_{i} \propto K_{i i}^{0.47(1)}$, a relationship reminiscent of a simple harmonic oscillator. Thus, while the total vibrational spectrum is set by the collective nature of the disordered network, the average vibrations of individual particles are determined primarily by local stiffness.

While these results explain properties of disordered clusters, it's natural to consider how they inform other commonly studied systems. Specifically, since the average number of nearest neighbors and mean confining stiffness correlate strongly with the median phonon frequency, our observations suggest that anytime the average number of nearest neighbors is increased in a disordered system, it is likely that the median frequency of the ensemble will increase as well. This effect should apply to different physical systems ranging from athermal jammed packings of purely repulsive particles ${ }^{4,5}$ to aging glasses ${ }^{15}$ and the observation suggests a spectroscopic means to probe average coordination and changes thereof.

Finally, the results presented here suggest that the vibrational modes can be shifted to lower (higher) energy by "doping" a glass with particles that are softer (stiffer) than average; these particles would also have especially low (high) average frequencies. This could be accomplished in colloids by mixing hard and soft particles, or by mixing particles with different amounts of surface charge. For atomic or molecular glass-formers, this could be accomplished by mixing socalled "fragile" glasses with "strong" glasses. ${ }^{20}$ Interestingly, recent works have suggested that particles in glasses that participate more than average in quasi-localized low frequency modes are more prone to rearrangement. ${ }^{21-24}$ Based on our findings, we speculate that regions containing soft "dopant" particles may be likely to rearrange. Alternatively, doping a glass with a small number of especially hard particles could potentially create regions that are unlikely to rearrange. This could potentially also be accomplished by creating regions 
with small $N N$, perhaps with laser tweezers, as previous studies found such regions to be associated with enhanced participation in quasi-localized modes..$^{25,26}$

\section{ACKNOWLEDGMENTS}

We thank Kevin Aptowicz, Piotr Habdas, Carl Goodrich, and Andrea Liu for helpful discussions, and we gratefully acknowledge financial support from the National Science Foundation through Grant Nos. DMR12-05463, the PENN MRSEC DMR11-20901, and NASA NNX08AO0G. Z.Z. gratefully acknowledges financial support from the National Basic Research Program of China through Grant No. 2012 CB821500.

\section{APPENDIX: DERIVATION OF RELATIONSHIP BETWEEN $\omega_{\text {Med }}$ AND $\overline{K_{i i}}$}

The strong relationship between $\bar{K}_{i i}$ and $\omega_{\text {Med }}$ can be understood from some simple linear algebra. The eigenvalues of the stiffness matrix are obtained from the relation $\left(K_{i j}-\omega^{2} I_{i j}\right) e_{\omega_{l}}=0$, where $e_{\omega_{l}}$ is the eigenvector for the $l$ th mode with frequency $\omega_{l}$ and $I_{i j}$ is the identity matrix. If we define a new matrix, $A_{i j}=K_{i j}-c I_{i j}$, where $c$ is a chosen scalar, we can then write $\left(K_{i j}-\omega_{l}^{2} I_{i j}\right) e_{\omega_{l}}=\left(A_{i j}+c I_{i j}\right.$ $\left.-\omega_{l}^{2} I_{i j}\right) e_{\omega_{l}}=\left(A_{i j}-\left[\omega_{l}^{2}-c\right] I_{i j}\right) e_{\omega_{l}}=\left(A_{i j}-\lambda_{l} I_{i j}\right) e_{\omega_{l}}$, where $\lambda_{l}$ is the $l$ th eigenvalue of $A_{i j}$ (just as $\omega_{l}^{2}$ is the $l$ th eigenvalue of $K_{i j}$. Thus, $\lambda_{l}=\omega_{l}^{2}-c$ and $\omega_{l}=\sqrt{\lambda_{l}+c}$. Note that while $\omega_{l}$ must be positive, $\lambda_{l}$ may be positive or negative, depending on the chosen value of $c$. However, what value should be selected for $c$ ?

The median of $\omega$ is the $N$ th mode (there are $2 N$ degrees of freedom), $\omega_{N}$, and $\omega_{N}=\sqrt{\lambda_{N}+c}$. If $c$ is chosen such that $c \approx \omega_{N}^{2}$, then $\lambda_{N}$ is small and $\omega_{N}$ only depends strongly on $c$. For example, if we set $c=\omega_{N}^{2}$, then $\omega_{N}=\sqrt{\lambda_{N}+c}$ $=\sqrt{\lambda_{N}+\omega_{N}^{2}}$, so $\lambda_{N}$ trivially is 0 .

In an attempt to identify an interesting or useful value for $c$, we next set $c=\bar{K}_{i i}$. We have already seen that $\bar{K}_{i i}$

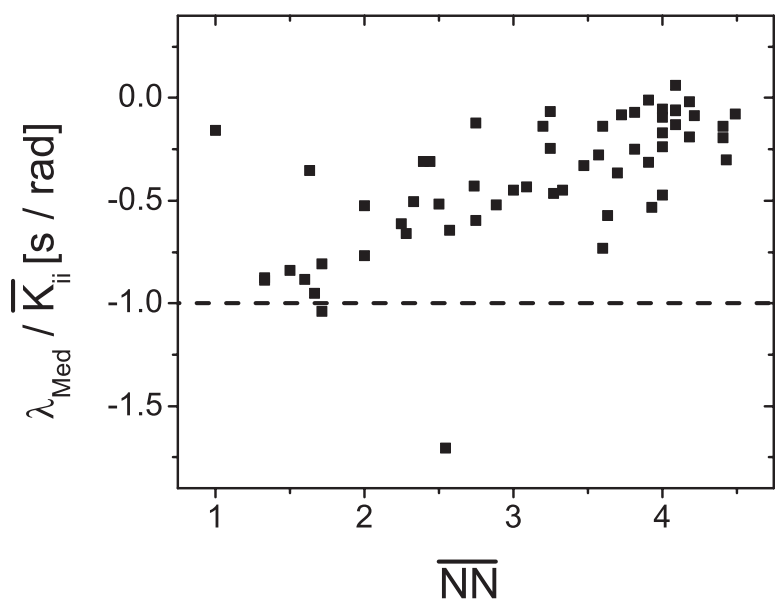

FIG. 7. The eigenvalues, $\lambda$, of matrix $A_{i j}=K_{i j}-\overline{K_{i i}} I_{i j}$, normalized by the mean of the diagonal value of the stiffness matrix, i.e., $\bar{K}_{i i}$, and plotted versus the number of neighbors $N N$. correlates with $\omega_{\text {Med }}$, making it a natural choice. Further, the best linear fit between $\sqrt{\bar{K}_{i i}}$ and $\omega_{\text {Med }}$ has a slope of nearly 1 (1.09(4)), implying that $\lambda_{N}$ is likely small compared to $\sqrt{\bar{K}_{i i}}$. Placing $c=\bar{K}_{i i}$ into the formula above gives us $\omega_{\text {Med }}=\sqrt{\lambda_{\text {Med }}+\bar{K}_{i i}} \cdot \lambda_{\text {Med }}$ can be calculated from the matrix $A_{i j}=K_{i j}-\bar{K}_{i i} I_{i j}$ (Fig. 7). There is little correlation between $\overline{N N}$ and $\lambda$, and $\lambda$ is smaller in magnitude than $\omega_{\text {Med }}^{2}$ (Fig. 7). This relationship should also be expected as Fig. 5 shows that $\bar{K}_{i i}$ is typically larger than $\omega_{\text {Med }}^{2}$, which, in turn, requires that $\lambda$ is negative.

While we have experimentally demonstrated that $\lambda_{\text {Med }}$ is small compared to $\overline{K_{i i}}$, could we have predicted this relationship based on the overall shape of $K_{i j}$ ? If $A_{i j}$ were a random matrix, its median eigenvalue would be 0 , according to the Wigner semi-circle law. ${ }^{27}$ Of course, $A_{i j}$ is not a truly random matrix. Thus, further study is required to elucidate the origin of the relationship between $\overline{K_{i i}}$ and $\omega_{\text {Med }}$.

${ }^{1}$ G. Meng, N. Arkus, M. P. Brenner, and V. N. Manoharan, Science 327, 560 (2010).

${ }^{2}$ P. J. Lu, J. C. Conrad, H. M. Wyss, A. B. Schofield, and D. A. Weitz, Phys. Rev. Lett. 96, 028306 (2006).

${ }^{3}$ P. J. Yunker, K. Chen, Z. Zhang, and A. G. Yodh, Phys. Rev. Lett. 106, 225503 (2011)

${ }^{4}$ C. S. O'Hern, S. A. Langer, A. J. Liu, and S. R. Nagel, Phys. Rev. Lett. 88, 075507 (2002)

${ }^{5}$ M. Wyart, S. R. Nagel, and T. A. Witten, EPL 72, 486 (2005).

${ }^{6}$ Z. Zhang, P. J. Yunker, P. Habdas, and A. G. Yodh, Phys. Rev. Lett. 107, 208303 (2011).

${ }^{7}$ D. Beysens and T. Narayanan, J Stat. Phys. 95, 997 (1999).

${ }^{8}$ C. Hertlein, L. Helden, A. Gambassi, S. Dietrich, and C. Bechinger, Nature (London) 451, 172 (2008).

${ }^{9}$ F. Soyka, O. Zvyagolskaya, C. Hertlein, L. Helden, and C. Bechinger, Phys. Rev. Lett. 101, 208301 (2008).

${ }^{10}$ A. Ghosh, R. Mari, V. Chikkadi, P. Schall, J. Kurchan, and D. Bonn, Soft Matter 6, 3082 (2010).

${ }^{11}$ K. Chen, W. G. Ellenbroek, Z. Zhang, D. T. N. Chen, P. J. Yunker, S. Henkes, C. Brito, O. Dauchot, W. van Saarloos, and A. J. Liu et al., Phys. Rev. Lett. 105, 025501 (2010).

${ }^{12}$ D. Kaya, N. L. Green, C. E. Maloney, and M. F. Islam, Science 329, 656 (2010).

${ }^{13}$ A. Ghosh, V. K. Chikkadi, P. Schall, J. Kurchan, and D. Bonn, Phys. Rev. Lett. 104, 248305 (2010).

${ }^{14}$ Z. Zhang, N. Xu, D. T. Chen, P. Yunker, A. M. Alsayed, K. B. Aptowicz, P. Habdas, A. J. Liu, S. R. Nagel, and A. G. Yodh, Nature (London) 459, 230 (2009).

${ }^{15}$ P. Yunker, Z. Zhang, K. B. Aptowicz, and A. G. Yodh, Phys. Rev. Lett. 103, 115701 (2009).

${ }^{16}$ P. Yunker, Z. Zhang, and A. G. Yodh, Phys. Rev. Lett. 104, 015701 (2010).

${ }^{17}$ D. G. Grier, Nature (London) 424, 810 (2003).

${ }^{18}$ C. Brito and M. Wyart, EPL 76, 149 (2006).

${ }^{19}$ K. Chen, T. Still, K. B. Aptowicz, S. Schoenholz, M. Schindler, A. C. Maggs, A. J. Liu, T. Lubensky, and A. G. Yodh, "Phonons in pristine and imperfect two-dimensional soft colloidal crystals," arXiv:1212.1741.

${ }^{20}$ C. A. Angell, Science 267, 1924 (1995).

${ }^{21}$ M. L. Manning and A. J. Liu, Phys. Rev. Lett. 107, 108302 (2011).

${ }^{22}$ K. Chen, M. L. Manning, P. J. Yunker, W. G. Ellenbroek, Z. Zhang, A. J. Liu, and A. G. Yodh, Phys. Rev. Lett. 107, 108301 (2011).

${ }^{23}$ A. Widmer-Cooper, H. Perry, P. Harrowell, and D. R. Reichman, Nat. Phys. 4, 711 (2008).

${ }^{24}$ A. Ghosh, V. Chikkadi, P. Schall, and D. Bonn, Phys. Rev. Lett. 107, 188303 (2011).

${ }^{25}$ H. Shintani and H. Tanaka, Nat. Mater. 7, 870 (2008).

${ }^{26}$ P. Tan, N. Xu, A. B. Schofield, and L. Xu, Phys. Rev. Lett. 108, 095501 (2012).

${ }^{27}$ E. P. Wigner, Ann. Math. 67, 325 (1958). 